\title{
LA BLOGOSFERA COMO MEDIO DE EVALUACIÓN FORMATIVA Y COMPARTIDA. UNA EXPERIENCIA EN LA FORMACIÓN INICIAL DEL PROFESORADO
}

The blogosphere as Formative and Shared Assessment tool. An experience in Initial Teacher Education

A blogosfera como um meio de avaliação formativa e compartilhada. Uma experiência na Formação Inicial de Professores

\begin{abstract}
Este estudio se ha llevado a cabo dentro del proyecto de I+D+i: "La competencias docentes en la formación inicial del profesorado de educación física”. Convocatoria de noviembre de 2013 del Programa Estatal de Investigación, Desarrollo e Innovación Orientada a los Retos de la Sociedad, en el marco del Plan Estatal de Investigación Científica y Técnica y de Innovación 2013-2016. Referencia: EDU 2013-42024-R. Duración: 3 años (2014-2016).
\end{abstract}

\section{Rodrigo Atienza Gago}

Universitat de València, España. Teléfono: +34 961625095. Correo electrónico: rodrigo.atienza@uv.es

\begin{abstract}
Resumen
Este documento presenta una experiencia de evaluación formativa a través de una blogosfera educativa (conjunto de blogs interconectados). En ella participaron 47 estudiantes de Formación Inicial de Profesorado de Educación Física. Esta experiencia trata de dar respuesta a la necesidad de proporcionar retroinformación de forma inmediata, involucrar al alumnado en el proceso de evaluación y de que este proceso sea lo más abierto, democrático y transparente posible. El desarrollo de la experiencia parte de un Proyecto de Aprendizaje Tutorado grupal, consistente en el diseño de una unidad didáctica de Educación Física y el desarrollo práctico de una de sus sesiones. Los contenidos de dicho proyecto se fueron colgando en un blog educativo generado por el alumnado. Tanto el profesor de la asignatura como el alumnado proporcionaron retroalimentación a través de comentarios en el blog. Los resultados obtenidos nos permiten ser optimistas, aunque todavía deben realizarse ajustes a la propuesta.

La blogosfera como medio de evaluación formativa y compartida. Una experiencia en la formación inicial del profesorado


Palabras clave: Evaluación formativa; Blogosfera; Educación superior; Educación física

\begin{abstract}
This paper presents an experience of formative assessment through an educational blogosphere (set of interconnected blogs). The study was conducted with a sample of 47 students from the Initial Formation of Physical Education Teachers. This experience tries to respond to the need to provide immediate feedback, to involve students in the assessment process and to make this process as open, democratic and transparent as possible. The development of the experience starts from a Project Oriented Learning (POL), consisting of the design of a didactic unit of Physical Education and the practical development of one of its sessions. The contents of this POL were hung in an educational blog generated by the students. Both the teacher and the students provided feedback through comments on the blog. The results obtained allow us to be optimistic, although adjustments must still be made to the proposal.
\end{abstract}

Keywords: Formative assessment; Blogosphere; Higher education; Physical education

\title{
Resumo
}

Este documento apresenta uma experiência de avaliação formativa através de uma blogosfera educacional (conjunto de blogs interconectados). Estiveram presentes 47 alunos de Formação Inicial de Professores de Educação Física. Esta experiência está a responder à necessidade de fornecer feedback imediato, envolver os alunos no processo de avaliação e que este processo é tão aberto, democrática e transparente possível. A experiência de desenvolvimento de um Projeto grupal de Aprendizagem Orientado que consiste em projetar uma unidade de ensino da educação física e desenvolvimento prático de uma das suas sessões. Os conteúdos deste projeto foram pendurados em um blog de educação gerado pelos alunos. Tanto o professor que os alunos forneceram feedback através de comentários no blog. Os resultados nos permitem ser otimistas, embora ajustes à proposta ainda precisa ser feito.

Palavras-chave: Avaliação formativa; Blogosfera; Educação superior; Educação física

La blogosfera como medio de evaluación formativa y compartida. Una experiencia en la formación inicial del profesorado 


\section{Introducción}

Un grupo de docentes de la Facultad de Magisterio de la Universitat de València venimos desarrollando propuestas de evaluación formativa y compartida (EFyC) en distintas asignaturas de Formación Inicial del Profesorado de Educación Física. A lo largo de estos años se han realizado distintas revisiones de nuestras experiencias, con el fin de mejorar las líneas maestras del sistema de evaluación (López-Pastor, 2017). Tras esta labor se han identificado una serie de limitaciones a las que consideramos oportuno dar respuesta, entre ellas destacamos:

- La dificultad encontrada para proporcionar una retroalimentación lo más inmediatamente posible.

- La dificultad encontrada para que la retroinformación proporcionada a un determinado grupo de trabajo contribuya al aprendizaje de toda la clase.

- La necesidad de fomentar la participación del alumnado en un proceso continuo de evaluación hacia sus compañeras y compañeros.

Para tratar de abordar estos retos se ha recurrido al entorno virtual, para ello diseñó una blogosfera ${ }^{1}$ como marco para la vertebración del proceso de EFyC. Con ello se intentó que los edublogs (blogs educativos) que formaron parte de dicha blogosfera nos permitieran ampliar los límites espaciotemporales del aula, facilitar la interacción y la participación del alumnado durante su proceso de aprendizaje (Antolín et al., 2011).

\section{Contextualización}

La experiencia que aquí se relata tuvo lugar durante el curso académico 2016/17, participando en ella un grupo de 47 estudiantes (cuyas características principales quedan reflejadas en la figura 1) de Grado de Maestra/o en Educación Primaria. Concretamente se desarrolló en la asignatura Didáctica de la Educación Física en la Educación Primaria, materia troncal de segundo curso que consta de 6 créditos ECTS de los cuales 2,4 son presenciales de gran grupo y 3,6 son no presenciales.

\footnotetext{
${ }^{1}$ El término blogosfera hace referencia al conjunto de blogs que están interconectados entre sí a través de una serie de enlaces, comentarios, históricos, referencias, etc. (Usabiaga-Arruabarrena, Martos-García, \& Valencia-Peris, 2014).

La blogosfera como medio de evaluación formativa y compartida. Una experiencia en la formación inicial del profesorado 


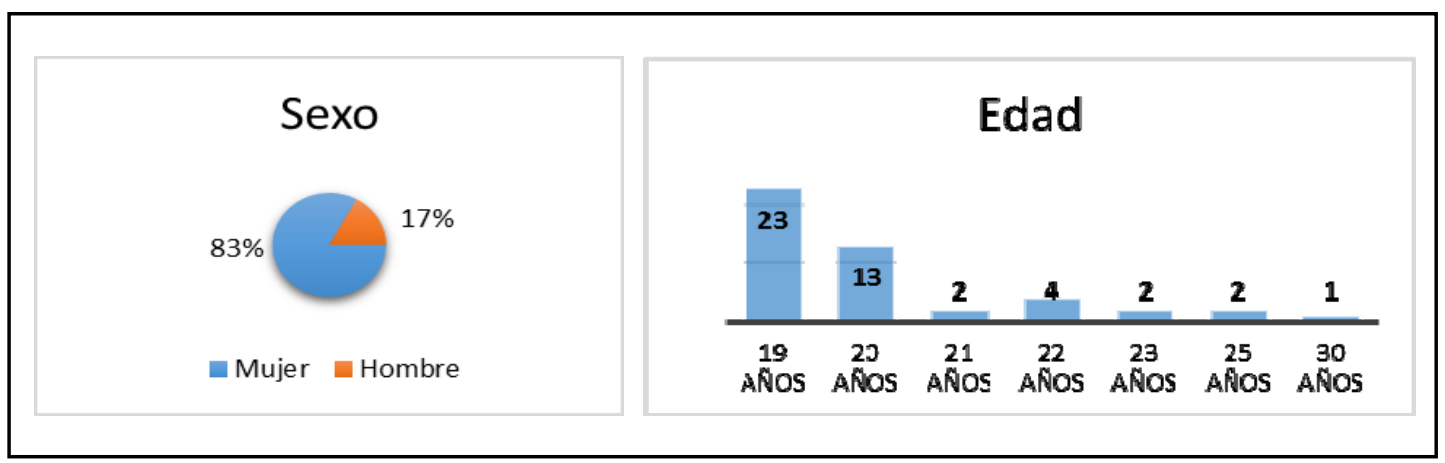

Figura 1.

Características de la muestra del estudio

\section{Diseño y desarrollo}

La principal actividad de aprendizaje que realiza el alumnado a lo largo de la asignatura es el diseño de una unidad didáctica (UD) de Educación Física para la Educación Primaria. Se trata de una tarea grupal teórico-práctica organizada en forma de proyecto de aprendizaje tutorado (PAT) (ver Manrique, López-Pastor, Monjas, \& Real, 2010).

A principio de curso se diseñó un edublog para la asignatura, en él se editaron los contenidos de la asignatura: información de carácter general, avisos, temario, etc. Por su parte, el alumnado -organizado en grupos de trabajo-, debía activar un blog grupal (que formaría parte de la blogosfera) en donde colgaría los distintos apartados de la UD que estaban diseñando: introducción, análisis del contexto, objetivos, competencias, contenidos, metodología, etc.

El profesor, proporcionó retroalimentación a través de comentarios en los distintos posts de los blogs grupales donde se desarrollaban las UUDD. Se ofrecieron una serie de consideraciones sobre aspectos desarrollados correctamente, apreciaciones sobre posibles errores e indicaciones que permitieran mejorar el trabajo final. Para ello se utilizó una escala de nivel consensuada con el alumnado. El tiempo estimado de retroalimentación fue de un día. El alumnado tuvo acceso tanto a los comentarios vertidos sobre su propia UD, como a las aportaciones a otros grupos. Cada grupo pudo rehacer los distintos apartados en nuevos posts de forma que se apreció la evolución del trabajo durante el proceso (por ejemplo: Metodología I, Metodología II, Metodología III, etc.).

La blogosfera como medio de evaluación formativa y compartida. Una experiencia en la formación inicial del profesorado 
Por otro lado, cada grupo de trabajo tuvo que dirigir ante sus compañeros y compañeras una de las sesiones prácticas diseñadas en su UD. Este proceso tuvo lugar en dos momentos (un ensayo a mitad de cuatrimestre y una versión definitiva a final de curso) de forma que entre uno y otro se produjo retroinformación por parte del profesorado y del alumnado. Dicha evaluación se promovió de forma presencial al finalizar la sesión y virtual a través de la blogosfera. Para tales efectos se utilizó una ficha de observación también consensuada a principio de curso.

\section{Evaluación y conclusiones}

Tras la puesta en práctica de la experiencia se han encontrado algunas ventajas, entre las que destacamos:

- La blogosfera permitió proporcionar retroinformación de forma casi inmediata a lo largo del proceso de aprendizaje.

- El alumnado no solo conoce los comentarios que el docente le aporta a su grupo a través del blog, sino que también puede acceder a los de otros grupos.

- El alumnado tiene la posibilidad de contribuir a la evaluación de sus compañeras y compañeros.

Por otro lado, se detectaron algunas limitaciones:

- La escasa implicación del alumnado a la hora de proporcionar retroinformación al resto de grupos a través de los medios establecidos. El alumnado optó por canales informales según su afinidad personal. Este intercambio de opiniones, a pesar de que pudiera ser válido y veraz, no era transparente para el resto de compañeras y compañeros ni para el docente.

- Parte del alumnado no está familiarizado con la el diseño y mantenimiento de blogs lo que dificultó en algunos casos la dinámica de la propuesta de evaluación planteada.

De cara a próximos cursos se proponen las siguientes medidas:

- Incentivar la participación del alumnado en las labores de evaluación a otros grupos bien estableciendo como requisito realizar un número determinado de aportaciones, bien reservando una parte de la calificación a estas labores.

La blogosfera como medio de evaluación formativa y compartida. Una experiencia en la formación inicial del profesorado 
- Hacer un sondeo diagnóstico para conocer cuántas personas tienen experiencias previas con blogs y así establecer tutorías técnicas de la web 2.0 que les ayuden a desenvolverse en dicho medio.

\section{Referencias}

Antolín, L., Molina, J.P., Villamón, M., Devís-Devís, J., \& Pérez-Samaniego, V. (2011). Uso de blogs en Ciencias de la Actividad Física y el Deporte. @tic revista d'innovació educativa, 7, 12-18.

López-Pastor, V.M. (2017). Evaluación formativa y compartida: evaluar para aprender y la implicación del alumnado en los procesos de evaluación y aprendizaje. En V. M. López-Pastor \& Á. Pérez-Pueyo (Coords.). Evaluación formativa y compartida en educación: experiencias de éxito en todas las etapas educativas (pp. 34-69). León: Universidad de León.

Manrique, J. C., López-Pastor, V.M., Monjas, R., \& Real, F. (2010). El potencial de los proyectos de aprendizaje tutorado y los sistemas de evaluación formativa en la mejora de la autonomía del alumnado. Una experiencia interdisciplinar en formación inicial del profesorado. Revista Española de Educación Física y Deportes, 14, 39-57.

Usabiaga-Arruabarrena, O., Martos-García, D., \& Valencia-Peris, A. (2014). Propuesta de innovación educativa para el futuro profesorado de educación física a través de una blogosfera. Revista Española de Educación Física y Deportes, 406, 85-92.

La blogosfera como medio de evaluación formativa y compartida. Una experiencia en la formación inicial del profesorado 